\title{
THREE DIMENSIONAL SIMILARITY SOLUTIONS OF THE NONLINEAR DIFFUSION EQUATION FROM OPTIMIZATION AND FIRST INTEGRALS
}

\author{
J.-Y. PARLANGE, R. D. BRADDOCK, G. SANDER AND F. STAGNITTI
}

(Received 11 June 1980)

(Revised 18 April 1981)

\begin{abstract}
For diffusion problems, the boundary conditions are specified at two distinct points, yielding a two end-point boundary value problem which normally requires iterative techniques. For spherical geometry, it is possible to specify the boundary conditions at the same points, approximately, by using an optimization principle for arbitrary diffusivity. When the diffusivity obeys a power or an exponential law, a first integral exists and iteration can be avoided. For those two exact cases, it is shown that the general optimization result is extremely accurate when diffusivity increases rapidly with concentration.
\end{abstract}

\section{Introduction}

The nonlinear diffusion equation

$$
\partial c / \partial t=\nabla \cdot(D \nabla c)
$$

occurs in a variety of scientific and technological fields, for example, hydrology, soil physics, metallurgy, chemical reactors, chromatography and the drying of grains, paper, wood, bricks, ceramics and so on. Hence there is the continuing interest in the understanding of the nature of its solutions. A general class of solutions, in particular, has been studied when only one geometrical dimension, $\xi$, enters the problem. In one dimension, $\xi$ is the distance from a plane source, or from a cylindrical or spherical source in two and three dimensions. For this general class and in three dimensions, (1.1) reduces to

$$
\partial c / \partial t=\xi^{-2} \partial\left(\xi^{2} D \partial c / \partial \xi\right) / \partial \xi
$$

@Copyright Australian Mathematical Society 1982 
We are interested in a similarity solution when only the independent variable $\phi=\xi t^{-\frac{1}{2}}$, enters the problem. The amount of material, $M=4 \pi \int_{0}^{\infty} c \xi^{2} d \xi$, is then proportional to $t^{3 / 2}$ at any time, and the flux of material entering the system $q=d M / d t$, is proportional to $t^{\frac{1}{2}}$. The present similarity solution implies that we can define a positive constant, $Q$, such that $q=4 \pi Q t^{\frac{1}{2}}$. Equation (1.2) then becomes

$$
-\frac{1}{2} \phi d c / d \phi=\phi^{-2} d\left[\phi^{2} D d c / d \phi\right] / d \phi
$$

and, by integration,

$$
D=-\frac{1}{2} \phi^{-2}(d \phi / d c) \int_{0}^{c} \phi^{3}(\bar{c}) d \bar{c},
$$

where concentrations are measured relative to the zero initial concentration and the latter is assumed to be uniform. Note that, since $d \phi / d c \rightarrow \infty$ as $t \rightarrow 0$, the lower limit in the integral entering (1.4) must be zero if $D$ is to remain finite.

As long as the diffusivity is bounded, it is also clear that, since $q$ is proportional to $t^{\frac{1}{2}}$, the concentration at $\xi=0$ would tend to increase without limit at $\phi=0$ if (1.4) held there. This of course cannot happen physically. Thus, we consider the case when $c$ has a maximum value called the saturated concentration. This saturated concentration is, by definition, taken as unity in the following. Hence there is some value, $\phi_{1}$, as yet unknown, such that (1.4) holds only for $\phi \geqslant \phi_{1}$, that is, in the unsaturated zone. In the saturated zone we have, by definition,

$$
c=1 \text { for } \phi \leqslant \phi_{1} \text {. }
$$

The value of $\phi_{1}$ must of course be related to the imposed flux.

Finally, $c$ satisfies equation (1.5) in the saturated zone and equation (1.4) or (1.3) in the unsaturated region, that is, for $\phi>\phi_{1}$. For a given $\phi_{1}$, the problem can be entirely solved with the conditions, $c=1$ at $\phi=\phi_{1}$ and $c \rightarrow 0$ as $\phi \rightarrow \infty$. However $Q$, rather than $\phi_{1}$, is normally given and those two quantities are related by overall conservation of mass, that is, applying equation (1.4) at $c=1$, or

$$
2 Q=\int_{0}^{1} \phi^{3} d c
$$

Hence, in principle, the problem is solved for an aribtrary $\phi_{1}$, yielding $\phi\left(c, \phi_{1}\right)$; then equation (1.6) allows us to relate $\phi_{1}$ and $Q$.

The experimental determination of $D(c)$ is often carried out by measuring $\phi(c)$ in one dimension, [4], [6], [3]. The two dimensional experiment has also been used for that purpose (for example, see [11] and [13]). The three dimensional equation (1.4), however, has apparently not been used in the past. It would, however, be the best choice when properties vary with position, as is 
often the case, for example, in soil physics, [2]. That is, one and two dimensional experiments involve diffusion from a plane and a line, while in three dimensions diffusion is around a point source. Also, the more limited extent of the source requires the use of less material, a useful consideration in field experiments. An extensive literature exists concerning the similarity solutions of the diffusion equation, that is, calculating $\phi$ when $D$ is given, in one, and to a lesser extent, in two dimensions (see, for example, [4] and [8]). We shall now study some properties of the solutions in three dimensions. First, an optimization principle is derived and its physical implications given. Then an exact class of solutions is obtained when (1.4) has a first integral. Comparison of the two results will finally illustrate the importance and validity of the optimization principle.

\section{Optimization}

It is straightforward to check that the following integral, $I$, is stationary when (1.4) holds

$$
I=\int_{0}^{1}\left\{4 D \ln \left|\frac{d \phi^{-1}}{d c}\right|-\phi^{2}\right\} d c
$$

that is, (1.4) is the Euler-Lagrange equation associated with (2.1). Of course, an optimization principle is useful only if the optimized parameter is needed in practice. Here the optimization principle will be useful if $\phi_{1}$ can be predicted accurately. Numerical integration of equation (1.4) can then be carried out easily for $\phi>\phi_{1}$ since, at $\phi=\phi_{1}, d \phi / d c$ is given by the imposed flux and $c=1$ from the saturation condition.

In one dimension a similar principle exists, [7] and [1], when the concentration at the surface $\xi=0$ is, by definition, taken as unity. It was shown that if $D$ varies rapidly with $c$, then the optimization principle leads to a boundary condition at $\phi=0$, which, together with $c=1$ at $\phi=0$, allows the direct numerical evaluation of the one-dimensional similarity solution. Without optimization, the condition $c \rightarrow 0$ as $\rho \rightarrow \infty$ must be used, which is not applied at the same point as the condition $c=1$ at $\phi=0$. Hence the solution is normally obtained by cumbersome numerical iterations when the optimization result is not used. The three dimensional case considered here follows a similar scenario.

As in one dimension, we expect that the optimization principle will be useful when $D$ increases rapidly with $c$. This is an important case, not only because standard linearization techniques do not apply, but also because it is physically 
relevant in many practical cases. For instance, water diffusion in soil is often represented, [10], by

$$
D=D_{1} \exp (\mu(c-1)),
$$

with $\mu$ taking values between 8 and 10. For this diffusivity, and in general for any rapidly increasing diffusivity, we define a small parameter $\varepsilon$, as,

$$
\varepsilon=\int_{0}^{1}(1-c) D d c /\left(\int_{0}^{1} D d c\right)
$$

The limit $\varepsilon \rightarrow 0$ corresponds to a diffusivity different from zero only when $c=1$; for that limit $\phi$ is then a constant, independent of $c$, and $\int_{0}^{c} \phi^{3} d \bar{c}$ in (1.4) is proportional to $c$. When $\varepsilon$ is small, rather than zero, this last integral will not be exactly proportional to $c$, but will have a mild dependence on $c$, compared to that of $D$. Let us define then a function $f(c)$, such that $f(1)=1$ and replace $\frac{1}{2} \int_{0}^{c} \phi^{3}(\bar{c}) d \bar{c}$ by $[f \lambda]^{-1}$ in (1.4) where $\lambda$ is an unknown constant.

Then from (1.4)

$$
\phi_{1}^{-1}-\phi^{-1}=\lambda \int_{c}^{1} f D d \bar{c} .
$$

If $f(c)$ were exact, that is, if we had

$$
f(c)=2 /\left[\lambda \int_{0}^{c} \phi^{3}(\bar{c}) d \bar{c}\right]
$$

$\lambda$ would be equal to $Q^{-1}$.

However, to apply the optimization principle, some approximate choice of $f$ can be made, for example, $f=c$, or $f=1$ and then $\lambda$ is determined for that choice of $f$ to optimize the estimate of $\phi_{1}$. The value of $\lambda$ will then differ from $Q^{-1}$. We shall see later that our results are essentially independent of $f$ and hence it is not necessary to replace $f$ by any explicit function. Replacing $\phi$ in (2.1) by its value in (2.5) and writing $d I / d \lambda=0$, yields,

$$
2 \int_{0}^{1} D d c=\int_{0}^{1}\left(\phi_{1}^{-1}-\phi^{-1}\right) \phi^{3} d c .
$$

Note that direct integration of (1.4) yields (2.6). However many other integral conditions could have been obtained from (1.4). Overall mass conservation could also be used by applying (1.4) at $c=1$. However all such conditions are not optimal and lead to inaccurate relations between $\phi_{1}$ and $Q$. That is, (2.6) will be the appropriate condition, because it is obtained from an optimization principle. Replacing $\phi$ by its expression in (2.4) yields $\lambda$. Let us rewrite (2.6) as

$$
2 \int_{0}^{1} D d c=\int_{0}^{1} \phi^{3} d c\left[\phi_{1}^{-1}-\phi_{0}^{-1}\right]+\int_{0}^{1}\left(\phi_{0}^{-1}-\phi^{-1}\right) \phi^{3} d c,
$$

where $\phi_{0}$ characterizes the wetting front position, for example, the value of $\phi$ for $c \simeq 0$. Note that the value of $\phi$ for $c=0$, strictly, could be infinite depending on 
the behaviour of $f D$ as $c \rightarrow 0$. However, if this difficulty arises it is easily removed either by defining $\phi_{0}$ for a value of $c$ small but non zero, or by taking $f=1$ to define a finite $\phi_{0}$ in (2.5). Whatever the definition of $\phi_{0}$, the second term in the right hand side of (2.7) is an order $\varepsilon$ smaller than the first. Hence, limiting ourselves to the first two orders in (2.7), the second term can be calculated to its lowest order only, or, see the Appendix,

$$
\int_{0}^{1} D d c \simeq\left(\phi_{1}^{-1}-\phi_{0}^{-1}\right) Q-\frac{1}{2}\left(\phi_{0}^{2}-\phi_{1}^{2}\right) \varepsilon .
$$

If, in (1.6), $\phi$ is replaced by $\phi_{0}-\left(\phi_{0}-\phi\right)$, we obtain, up to the second order,

$$
2 Q \simeq \phi_{0}^{3}\left\{1+\left[\phi_{1} / \phi_{0}+\frac{1}{2} \phi_{1}^{2} / \phi_{0}^{2}+\ln \left(\phi_{1} / \phi_{0}\right)-\frac{3}{2}\right] \varepsilon\right\}
$$

Eliminating $\phi_{0}$ between (2.8) and (2.9) allows us to find $\phi_{1}$ for a given soil when $Q$ is given, independently of the exact choice for $f$ and $\phi_{0}$. This was of course the aim of the optimization method; in addition, note that $\phi_{0}$ is only an intermediary in the calculations so that its exact definition is irrelevant. It must also be remembered that (2.8) and (2.9) are approximate, that is, terms of order $O\left(\varepsilon^{2}\right)$, were ignored. In the next paragraph we shall assess the precision of these relations for two general classes of soils, by comparison with the exact solutions. These exact solutions are now found when first integrals to (1.4) exist.

\section{First integrals}

First integrals of (1.3) exist when $D$ belongs to certain classes of functions. A general method discussed in [5] is followed here. Setting

$$
u=\phi / D^{\frac{1}{2}}
$$

and

$$
w=\frac{1}{2} u+D d u / d D
$$

(1.4) becomes,

$$
\frac{1}{2} u^{3}+(w-u / 2) d\left(u^{2} w^{-1}\right) / d u+u^{2} w^{-1}\left[D^{-3 / 2} d\left(D^{5 / 2} d c / d D\right) / d c\right]=0,
$$

which represents a first integral if the term in the square brackets is constant. Or, by integration,

$$
d D / d c=D /\left[\mu+\nu^{-1} c\right]
$$


where $\mu$ and $\nu$ are arbitrary constants. Two general classes of solutions result from (3.4). If $\nu^{-1}=0$, then $D$ is of the form,

$$
D=D_{1} \exp \mu(c-1),
$$

where $D_{1}$ is a constant and $\mu>0$. If $\nu^{-1} \neq 0$, then $D$ is proportional to $\left(\mu+\nu^{-1} c\right)^{\nu}$. Taking $\left(\mu+\nu^{-1} c\right)$ as the new concentration, which is equivalent to taking $\mu=0$ without loss of generality, then

$$
D=D_{1} c^{\nu}
$$

where $D_{1}$ is a constant, and $\nu>0$. Those two classes of solutions, corresponding to the diffusivity obeying an exponential and a power law for arbitrary exponent will allow us, in particular, to check the validity of the optimization result. Equation (3.3) becomes

$$
2+\frac{1}{2} u^{2}+u w^{-1}\left(\frac{1}{2}+\nu^{-1}\right)+\left(w-\frac{1}{2} u\right) u d w^{-1} / d u=0 .
$$

Note the interesting result, which we shall use later, that $\mu$ does not enter in (3.7), hence the differential equation is independent of the diffusivity when the exponential law holds. Let us consider the behaviour of the solutions as $c \rightarrow 0$, that is, $u \rightarrow \infty$. For large $u, \nu^{-1} \neq 0$, we have by a straightforward expansion

$$
w^{-1}=-\frac{1}{2} \nu u-3 u^{-1} /\left(1+\nu^{-1}\right)+12 u^{-3} /\left(\left(2+\nu^{-1}\right)(\nu+1)\right)+O\left(u^{-5}\right),(3.8
$$

which shows that as $c \rightarrow 0, \phi$ is finite while $d c / d \phi$ is infinite, as expected physically since $D(0)=0$. On the other hand, for the exponential case, $D(0) \neq$ 0 , hence, as $c \rightarrow 0, \phi \rightarrow \infty$ and $d c / d \phi \rightarrow 0$. Then, (3.7) shows that

$$
w^{-1} \simeq C \exp \left(-u^{2} / 4\right) \quad \text { as } u \rightarrow \infty,
$$

where the constant, $C$, is a priori unknown, that is, it depends on the particular problem being solved.

Because of their different behaviour as $u \rightarrow \infty$, the solutions for power and exponential diffusivities are now considered separately.

\section{Power law}

Numerical integration of (3.7) was carried out using a fourth order standard Runge-Kutta integration. The starting point was taken for $u=50$ which is large enough for the third term in (3.8) to be negligible. Hence (3.8) could be used reliably to calculate $w$ corresponding to $u=50$. 
TABLE 1

Exact positions of the saturated front $\phi_{1} / D_{1}^{1 / 2}$ obtained by numerical integration of (3.7) when $D=D_{1} c^{\nu}$, for various values of $\nu$, and for various values of the flux $Q / D_{1}^{3 / 2}$.

\begin{tabular}{cccccc}
\hline$Q / D_{1}^{3 / 2}$ & $\nu=10$ & $\nu=8$ & $\nu=6$ & $\nu=4$ & $\nu=2$ \\
\hline 2.0 & 1.4808338 & 1.4592754 & 1.4267657 & 1.3723834 & 1.2635879 \\
1.9 & 1.4523489 & 1.4305360 & 1.3976871 & 1.3428529 & 1.2335782 \\
1.8 & 1.4228011 & 1.4007208 & 1.3675179 & 1.3122182 & 1.2024748 \\
1.7 & 1.3920876 & 1.3697257 & 1.3361530 & 1.2803743 & 1.1701750 \\
1.6 & 1.3600886 & 1.3374297 & 1.3034701 & 1.2471991 & 1.1365647 \\
1.5 & 1.3266635 & 1.3036908 & 1.2693261 & 1.1212549 & 1.1101508 \\
1.4 & 1.2916407 & 1.2683408 & 1.2335517 & 1.1762581 & 1.0648468 \\
1.3 & 1.2548360 & 1.2311784 & 1.1959443 & 1.1381235 & 1.0263932 \\
1.2 & 1.2159920 & 1.1919592 & 1.1562586 & 1.0979032 & 0.9859221 \\
1.1 & 1.1748156 & 1.1503823 & 1.1141929 & 1.0553006 & 0.9431625 \\
1.0 & 1.1309310 & 1.1060708 & 1.0693703 & 1.0099464 & 0.8977768 \\
0.9 & 1.0838608 & 1.1058542 & 1.0213103 & 0.9613723 & 0.8493449 \\
0.8 & 1.0329739 & 1.0071672 & 0.9693853 & 0.8970519 & 0.7973267 \\
0.7 & 0.9774199 & 0.9510921 & 0.9127519 & 0.8519303 & 0.7410164 \\
0.6 & 0.9160031 & 0.8889123 & 0.8502344 & 0.7891327 & 0.6794563 \\
0.5 & 0.8469585 & 0.8195084 & 0.7801161 & 0.7189625 & 0.6112989 \\
0.4 & 0.7675036 & 0.7394947 & 0.6997293 & 0.6389495 & 0.5345466 \\
0.3 & 0.6728179 & 0.6443552 & 0.6045473 & 0.5449907 & 0.4460187 \\
0.2 & 0.5532310 & 0.5247603 & 0.4857765 & 0.4293668 & 0.3400854 \\
0.1 & 0.3833401 & 0.3565894 & 0.3216377 & 0.2739800 & 0.2048061 \\
0.09 & 0.3608949 & 0.3348183 & 0.3007277 & 0.2546835 & 0.1887288 \\
0.08 & 0.3373072 & 0.3116100 & 0.2785485 & 0.2343679 & 0.1720063 \\
0.07 & 0.3116775 & 0.2867135 & 0.2548949 & 0.2128875 & 0.1545662 \\
0.06 & 0.2838301 & 0.2597988 & 0.2295016 & 0.1900584 & 0.1363200 \\
0.05 & 0.2532406 & 0.2304177 & 0.2020153 & 0.1656420 & 0.1171582 \\
0.04 & 0.2191517 & 0.1979341 & 0.1719455 & 0.1393181 & 9.0969110 \\
0.03 & 0.1803966 & 0.1613891 & 0.1385743 & 0.1106352 & 0.0748304 \\
0.02 & 0.1349962 & 0.1192031 & 0.1007608 & 0.0789105 & 0.0525229 \\
0.01 & 0.0789912 & 0.0683425 & 0.0564222 & 0.0429743 & 0.0265170 \\
0.001 & 0.0101762 & 0.0084537 & 0.0066782 & 0.0048465 & - \\
& & & & & \\
\hline
\end{tabular}

Table 1 gives values of $u_{1}=\phi_{1} / D_{1}^{1 / 2}$ for various values of $\nu$ and $Q / D_{1}^{3 / 2}$ where, from the definition of the flux, saturation is obtained from

$$
Q / D_{1}^{3 / 2}=w_{1}^{-1} \nu^{-1} u_{1}^{2} \text {. }
$$


Table 2 gives the corresponding results from the optimization procedure. In the present case $\varepsilon=1 /(\nu+2)$ and $\int_{0}^{1} D d c=D_{1} /(\nu+1)$ in (2.8) and (2.9).

\section{TABLE 2}

Positions of the saturated front $\phi_{1} / D_{1}^{3 / 2}$ obtained by optimization from (2.8) and (2.9), when $D=D_{1} c^{\nu}$, for various values of $\nu$ and $Q / D_{1}^{3 / 2}$.

\begin{tabular}{cccccc}
\hline$Q / D_{i}^{3 / 2}$ & $\nu=10$ & $\nu=8$ & $\nu=6$ & $\nu=4$ & $\nu=2$ \\
\hline 2.0 & 1.4808359 & 1.4592042 & 1.4265946 & 1.3718601 & 1.2611401 \\
1.9 & 1.4523487 & 1.4304620 & 1.3975102 & 1.3423149 & 1.2310921 \\
1.8 & 1.4227985 & 1.4006438 & 1.3673347 & 1.3116647 & 1.1999495 \\
1.7 & 1.3920823 & 1.3696456 & 1.3359630 & 1.2798044 & 1.1676118 \\
1.6 & 1.3600804 & 1.3373461 & 1.3032728 & 1.2466118 & 1.1339632 \\
1.5 & 1.3266523 & 1.3036037 & 1.2691209 & 1.2119441 & 1.0988691 \\
1.4 & 1.2916315 & 1.2682493 & 1.2333379 & 1.1756330 & 1.0621716 \\
1.3 & 1.2548183 & 1.2310823 & 1.1957212 & 1.1137477 & 1.0238416 \\
1.2 & 1.2159704 & 1.1918580 & 1.1560259 & 1.0972355 & 0.9831830 \\
1.1 & 1.1747893 & 1.1502762 & 1.1113948 & 1.0546099 & 1.9403970 \\
1.0 & 1.1309002 & 1.1059575 & 1.0691136 & 1.0092315 & 0.8949927 \\
0.9 & 1.0838238 & 1.0584223 & 1.0210400 & 0.9606323 & 0.8455211 \\
0.8 & 1.0329306 & 1.0070179 & 1.9691002 & 1.9082990 & 1.7945409 \\
0.7 & 0.9773695 & 0.9509540 & 0.9124504 & 0.8511395 & 0.7382588 \\
0.6 & 0.9159443 & 0.8889751 & 0.8499151 & 0.7883184 & 0.6767591 \\
0.5 & 0.8468899 & 0.8193472 & 0.7797780 & 0.7181299 & 0.6070833 \\
0.4 & 0.7674232 & 0.7393194 & 0.6993725 & 0.6381099 & 0.5303895 \\
0.3 & 0.6727236 & 0.6441648 & 0.6041755 & 0.5437246 & 0.4420654 \\
0.2 & 0.5531212 & 0.5245232 & 0.4852155 & 0.4280974 & 0.3366500 \\
0.1 & 0.3831606 & 0.3563986 & 0.3210816 & 0.2729017 & 0.2027049 \\
0.09 & 0.3609173 & 0.3346331 & 0.3004364 & 0.2536536 & 0.1868554 \\
0.09 & 0.3371233 & 0.3114322 & 0.2780180 & 0.2333969 & 0.1703902 \\
0.07 & 0.3114928 & 0.2865452 & 0.2543848 & 0.2119883 & 0.1532412 \\
0.06 & 0.2836463 & 0.2596428 & 0.2290197 & 0.1892475 & 0.1353265 \\
0.05 & 0.2530604 & 0.2302777 & 0.2015719 & 0.1649407 & 0.1165447 \\
0.04 & 0.2189795 & 0.1976744 & 0.1715555 & 0.1387542 & 0.0967665 \\
0.03 & 0.1802401 & 0.1611643 & 0.1382593 & 0.1102462 & 0.0758198 \\
0.02 & 0.1348695 & 0.1190348 & 0.1005545 & 0.0787470 & 0.0534604 \\
0.01 & 0.0789235 & 0.0682709 & 0.0563772 & 0.0430991 & 0.0293046 \\
0.001 & 0.0102073 & 0.0085093 & 0.0067903 & 0.0051454 & - \\
& & & & &
\end{tabular}

Eliminating $Q$ in (2.8), using its value in (2.9), gives $\phi_{0}$ for given $\phi_{1} / \phi_{0}$. This expression can then be used to eliminate $\phi_{0}^{3}$ in (2.9), resulting in a relationship 
between $Q$ and $\phi_{1} / \phi_{0}$ only. Hence for given $Q, \phi_{1} / \phi_{0}$ is easily calculated, then $\phi_{0}$ and finally $\phi_{1}$. The results are shown in Table 2 , for the same values of $\nu$ and $Q / D_{1}^{3 / 2}$ as in Table 1. Comparison of the two tables show the remarkable agreement between the exact values of Table 1 and the approximate values obtained by optimization in Table 2 . As expected, the agreement is better as $\nu$ increases. Even for $\nu$ as low as 2, the error is at most $1 \%$ if $Q / D_{1}^{3 / 2}$ is not too small, for example, larger than about 0.03 . This ensures that the saturated region contains $1 \%$ or more of the total amount of material. For vanishing fluxes, the saturated zone is relatively small and its extent more difficult to predict by optimization and the more so for lower values of $\nu$. When $Q / D_{1}^{3 / 2}$ is as large as 2 , or larger, the saturated zone contains a sizeable fraction of the total amount of material, and the approximate result is numerically very accurate, and again the accuracy increases with increasing $\nu$.

The two tables show explicitly the precision of the optimization procedure when the diffusivity obeys a power law. Furthermore, they may also be used to obtain some estimate of the expected error when $D$ does not exactly obey a power law. Then, for $\varepsilon$ given by (2.3), an equivalent $\nu$ is obtained from $\varepsilon=1 /(\nu+2)$. If the error then deduced from Tables 1 and 2 is acceptable, (2.8) and (2.9) can be used to calculate $\phi_{1}$ for an arbitrary $D$ and a given $Q$. Knowing $\phi_{1}$ and $Q$ then allows the straightforward numerical integration of (1.4) without cumbersome iteration.

We should also mention that when $D$ obeys a power law, (1.2) can be reduced to a form similar to (1.4) when $q$ is proportional to any power of time. This particular case, which has been studied for the one dimensional case [9], [12], can be quite useful in practice. However, contrary to the case studied here and represented by (1.4), it cannot be extended to an arbitrary diffusivity.

\section{Exponential law}

When the diffusivity obeys (2.2) the optimization procedure remains as before and (2.8) and (2.9) yield $u_{1}$ and $w_{1}$ when $Q / D_{1}^{3 / 2}$ and $\mu$ are given. As already mentioned, (3.7) is independent of the diffusivity in that case. It is then possible to solve (3.7) numerically, again by a fourth order Runge-Kutta method, with the initial point $\left(u_{1}, w_{1}\right)$ obtained by optimization. Then (3.2) can be used to obtain the corresponding exponent of the diffusivity, $\mu^{*}$, as

$$
\mu^{*}=\int_{u_{1}}^{\infty}\left(\frac{1}{2} u-w\right) d u
$$

where the integration is carried out numerically. Clearly $\mu^{*}$ will be different from $\mu$, the exponent of the diffusivity used in the optimization procedure to 
calculate the initial point $\left(u_{1}, w_{1}\right)$. Indeed, the difference between $\mu$ and $\mu^{*}$ gives a convenient measure of the error in the optimization procedure.

TABLE 3

Exact values of $\mu^{*}$ when $D=D_{1} \exp \left(\mu^{*}(c-1)\right)$ obtained numerically for given values of the flux, $Q / D_{1}^{3 / 2}$, and saturated front positions. The latter are chosen to be the same front positions given by optimization from (2.8) and (2.9), when $D$ is taken as $D_{1} \exp (\mu(c-1))$.

\begin{tabular}{cccccc}
\hline$Q / D_{1}^{3 / 2}$ & $\mu=10$ & $\mu=8$ & $\mu=6$ & $\mu=4$ & $\mu=2$ \\
\hline 2.0 & 10.000743 & 8.001718 & 6.004004 & 4.013487 & 2.037667 \\
1.8 & 10.000853 & 8.001743 & 6.004810 & 4.014935 & 2.040362 \\
1.6 & 10.001011 & 8.002224 & 6.005389 & 4.016658 & 2.043760 \\
1.4 & 10.001210 & 8.002589 & 6.006202 & 4.018854 & 2.047042 \\
1.2 & 10.001477 & 8.002070 & 6.007268 & 4.021655 & 2.051222 \\
1.0 & 10.001843 & 8.003740 & 6.008726 & 4.025350 & 2.056140 \\
0.8 & 10.002398 & 8.004724 & 6.010831 & 4.030438 & 2.061943 \\
0.6 & 10.003297 & 8.006307 & 6.014113 & 4.037917 & 2.068677 \\
0.7 & 10.005004 & 8.009227 & 6.019921 & 4.049839 & 2.074660 \\
0.2 & 10.009360 & 8.016319 & 6.032980 & 4.072181 & 2.076580 \\
0.18 & 10.010200 & 8.017624 & 6.035258 & 4.075525 & 2.075164 \\
0.16 & 10.011189 & 8.010151 & 6.037876 & 4.077145 & 2.073034 \\
0.14 & 10.012369 & 8.020960 & 6.040916 & 4.083065 & 2.069900 \\
0.12 & 10.013819 & 8.023133 & 6.044499 & 4.087320 & 2.065301 \\
0.10 & 10.015631 & 8.025801 & 6.048802 & 4.091853 & 2.058456 \\
0.08 & 10.017975 & 8.029203 & 6.054082 & 4.096496 & 2.047899 \\
0.06 & 10.021137 & 8.033664 & 6.060817 & 4.100688 & 2.030381 \\
0.04 & 10.025698 & 8.039918 & 6.069649 & 4.102446 & 1.995494 \\
0.02 & 10.033079 & 8.049814 & 6.082204 & 4.091899 & -
\end{tabular}

Table 3 gives $\mu^{*}$ for various values of $Q / D_{1}^{3 / 2}$ and $\mu$. As in the previous case, the error increases when $Q$ and $\mu$ decrease. Again this table could be used to estimate the error of optimization when $D$ does not obey an exponential law when $\mu \simeq 1 / \varepsilon$, with $\varepsilon$ calculated from(2.3). To compare the errors predicted in Tables 1 and 2 and Table 3, note that for the same $\varepsilon, \mu \simeq \nu+2$, and that the relative error in $\phi_{1}$ predicted by optimization is of the order of $\left(\mu^{*}-\mu\right) / \mu^{2}$. Under those conditions, both predict errors of the same order of magnitude for the same $Q / D_{1}^{3 / 2}$ and $\varepsilon$. This suggests that prediction of the optimization error when $D$ does not obey a power or an exponential law will be adequate. However, use of Tables 1 and 2 which give directly the error on $\phi_{1}$ is preferable in practice. 


\section{Summary}

In summary, we have developed a general optimization procedure which predicts, approximately, the position of the saturated front for a three dimensional diffusion problem. Knowledge of this position permits the straightforward integration of the diffusion equation. An estimate of the optimization error can also be obtained from exact results for special diffusivity functions. The optimization result can be used with confidence when the diffusivity increases rapidly with concentration, near saturation. It is also found that the error is less for large fluxes at the source.

\section{Acknowledgement}

This paper was prepared with the support of URG No. 35-0600-151 and ARGC No. F7-915139 I.

\section{Appendix: Derivation of (2.8) and (2.9)}

In the calculations, we limit ourselves to the main term and one correction of order $\varepsilon$, that is, we neglect higher order terms. In (2.7) we have

$$
\int_{0}^{1} \phi^{3} d c\left[\phi_{1}^{-1}-\phi_{0}^{-1}\right]=2 Q\left[\phi_{1}^{-1}-\phi_{0}^{-1}\right]
$$

the other integral is obviously of a smaller order, that is, it is a correction of order $\varepsilon$, hence we have to calculate it to its lower order only. Using (2.5) we can write,

$$
\int_{0}^{1}\left(\phi_{0}^{-1}-\phi^{-1}\right) \phi^{3} d c=\int_{0}^{1} \lambda \int_{0}^{c} f D d \bar{c}\left[\phi_{1}^{-1}-\lambda \int_{c}^{1} f D d \bar{c}\right]^{-3} d c .
$$

We now recognise that, to the lowest order, which is all we are interested in, and for $c$ near one, where the main contribution to the integral takes place, since $D$ increases rapidly with $c$, then

$$
\int_{0}^{c} f D d \bar{c} \simeq \alpha f D
$$

where $\alpha$ is a constant.

Note that, to the lowest order, we could also replace $f$ by one in (A2) and (A3). We then obtain

$$
\int_{0}^{1}\left(\phi_{0}^{-1}-\phi^{-1}\right) \phi^{3} d c \simeq \alpha \int_{0}^{1} \lambda f D\left[\phi_{1}^{-1}-\lambda \int_{c}^{1} f D d \bar{c}\right]^{-3} d c
$$


or,

$$
\int_{0}^{1}\left(\phi_{0}^{-1}-\phi^{-1}\right) \phi^{3} d c \simeq \frac{1}{2} \alpha\left[\phi_{1}^{2}-\phi_{0}^{2}\right]
$$

An estimate of $\alpha$ is obtained by integration of (A3) for $f=1$, that is, to the lowest order, yielding

$$
\alpha \simeq \int_{0}^{1}(1-c) D d c /\left(\int_{0}^{1} D d c\right)
$$

which shows that $\alpha$ is equal to $\varepsilon$, as defined in (2.3). Using (A1) and (A5) to eliminate the integrals in (2.7) yields (2.8).

The derivation of (2.9) proceeds along the same lines. Starting from (1.6) we have to estimate

$$
\int_{0}^{1} \phi^{3} d c=\phi_{0}^{3}+\int_{0}^{1}\left(\phi^{3}-\phi_{0}^{3}\right) d c,
$$

where the second integral is an order of magnitude smaller than $\phi_{0}^{3}$ and hence has to be calculated to its lowest order only. Using (A3) and remembering that $\alpha=\varepsilon$, we have

$$
\int_{0}^{1}\left(\phi^{3}-\phi_{0}^{3}\right) d c=-\varepsilon \int_{0}^{\theta} \phi_{0}^{3}(z)\left[\left(z^{2}+3 z+3\right) /(1+z)^{3}\right] d z,
$$

where $\theta=\phi_{0} / \phi_{1}-1$. By elementary integration, (A8) yields (2.9).

\section{References}

[1] N. Anderson and A. M. Arthurs, "Dual extremum principles for a nonlinear diffusion problem", Quart. Appl. Math. 35 (1977), 188-190.

[2] J. W. Biggar and D. R. Nielsen, "Spatial variability of the leaching characteristics of a field soil", Water Resour. Res. 12 (1976), 78-84.

[3] R. R. Bruce and A. Klute, "The measurement of soil-water diffusivity", Soil Sci. Soc. Am. Proc. 20 (1956), 458-462.

[4] J. Crank, The mathematics of diffusion (Oxford University Press, 1st Edition, 1956, Chapter XI).

[5] E. L. Ince, Ordinary differential equations (Dover, Ist Edition, 1956, Chapter IV).

[6] C. Matano, "On the relation between the diffusion coefficients and concentration of solid materials (the nickel-copper system)", Jap. J. Phys. 8 (1933), 109-113.

[7] J.-Y. Parlange, "On solving the flow equation in unsaturated soils by optimization: horizontal infiltration", Soil Sci. Soc. Am. Proc. 39 (1975), 415-418.

[8] J.-Y. Parlange, "Water transport in soils", Ann. Rev. Fluid. Mech. 12 (1980), 77-102.

[9] G. J. Pert, "A class of similar solutions of the nonlinear diffusion equation", J. Phys. A: Math. Gen. 10 (1977), 583-593.

[10] K. Reichardt, D. R. Neilsen and J. W. Biggar, "Scaling of horizontal infiltration into homogeneous soils", Soil Sci. Soc. Am. J. 36 (1977), 241-245.

[11] B. L. Sawhney and J.-Y. Parlange, "Radial movement of saturated zone under constant flux: Theory and application to the determination of soil-water diffusivity", Soil Sci. Soc. Am. J. 40 (1976), 635-639. 
[12] R. E. Smith and J.-Y. Parlange, "Optimal prediction of ponding", Trans. ASAE 20 (1977), 493-496.

[13] N. C. Turner and J.-Y. Parlange, "Two-dimensional similarity solution: Theory and application to the determination of soil-water diffusivity", Soil Sci. Soc. Am. Proc. 39 (1975), 387-390.

School of Australian Environmental Studies

Griffith University

Nathan

Queensland 4111 